\title{
Article \\ Nonexistence of Global Solutions to Higher-Order Time-Fractional Evolution Inequalities with Subcritical Degeneracy
}

\author{
Ravi P. Agarwal ${ }^{1,+} \mathbb{D}$, Soha Mohammad Alhumayan ${ }^{2,+}$, Mohamed Jleli ${ }^{2,+} \mathbb{D}$ and Bessem Samet ${ }^{2, *,+}(\mathbb{D}$ \\ 1 Department of Mathematics, Texas A \& M University-Kingsville, Kingsville, TX 78363, USA; \\ Ravi.Agarwal@tamuk.edu \\ 2 Department of Mathematics, College of Science, King Saud University, P.O. Box 2455, \\ Riyadh 11451, Saudi Arabia; s.alhumayan@gmail.com (S.M.A.); jleli@ksu.edu.sa (M.J.) \\ * Correspondence: bsamet@ksu.edu.sa \\ + These authors contributed equally to this work.
}

check for

updates

Citation: Agarwal, R.P.; Alhumayan, S.M.; Jleli, M.; Samet, B. Nonexistence of Global Solutions to Higher-Order Time-Fractional Evolution Inequalities with Subcritical Degeneracy. Mathematics 2021, 9, 2765. https://doi.org/10.3390/ math9212765

Academic Editor: Dong Yun Shin

Received: 7 October 2021

Accepted: 29 October 2021

Published: 31 October 2021

Publisher's Note: MDPI stays neutral with regard to jurisdictional claims in published maps and institutional affiliations.

Copyright: (c) 2021 by the authors. Licensee MDPI, Basel, Switzerland. This article is an open access article distributed under the terms and conditions of the Creative Commons Attribution (CC BY) license (https:// creativecommons.org/licenses/by/ $4.0 /)$.

\begin{abstract}
In this paper, we study the nonexistence of global weak solutions to higher-order timefractional evolution inequalities with subcritical degeneracy. Using the test function method and some integral estimates, we establish sufficient conditions depending on the parameters of the problems so that global weak solutions cannot exist globally.
\end{abstract}

Keywords: fractional-in-time evolution inequalities; subcritical degeneracy; global weak solutions; nonexistence

MSC: 35B44; 35B33; 26A33

\section{Introduction}

We are first concerned with the study of the nonexistence of global weak solutions to time-fractional evolution inequalities of the form:

$$
\frac{\partial^{\alpha} u}{\partial t^{\alpha}}(t, x)-|x|^{v} \Delta u(t, x) \geq|u(t, x)|^{p}, \quad(t, x) \in(0, \infty) \times \mathbb{R}^{N},
$$

subject to the initial conditions:

$$
\frac{\partial^{i} u}{\partial t^{i}}(0, x)=u_{i}(x), \quad i=0,1, \cdots, k-1, \quad x \in \mathbb{R}^{N}
$$

where $N \geq 1, k \geq 1$ is a natural number, $k-1<\alpha<k$, $\frac{\partial^{\alpha}}{\partial t^{\alpha}}$ is the time-Caputo fractional derivative of order $\alpha, p>1, v<\min \{2, N\}$, and $u_{i}|x|^{-v} \in L_{l o c}^{1}\left(\mathbb{R}^{N}\right)$ for all $i=0,1, \cdots, k-1$. Then, we consider the inhomogeneous problem:

$$
\frac{\partial^{\alpha} u}{\partial t^{\alpha}}(t, x)-|x|^{v} \Delta u(t, x) \geq|u(t, x)|^{p}+t^{\sigma} F(x), \quad(t, x) \in(0, \infty) \times \mathbb{R}^{N},
$$

subject to the initial condition (2), where $N, k \geq 1, k-1<\alpha<k, p>1, v<\min \{2, N\}$, $F|x|^{-v} \in L_{l o c}^{1}\left(\mathbb{R}^{N}\right), \sigma>-1$, and $u_{i}|x|^{-v} \in L_{l o c}^{1}\left(\mathbb{R}^{N}\right)$ for all $i=0,1, \cdots, k-1$.

In the limited case $\alpha \downarrow k,(1)$ reduces to the differential inequality:

$$
\frac{\partial^{k} u}{\partial t^{k}}(t, x)-|x|^{v} \Delta u(t, x) \geq|u(t, x)|^{p}
$$

When $k=1$, Mitidieri and Pohozaev [1] studied (2)-(4) in both cases $v=2$ (critical degeneracy) and $v<2$ (subcritical degeneracy). In the case where $v=2$, it was shown that 
under suitable conditions on the initial value $u_{0}$, if $1<p \leq 3$, then (2)-(4) has no global weak solutions. In the case $v<\min \{2, N\}$, it was shown that under suitable conditions on the initial value $u_{0}$, if $1<p \leq 1+\frac{2-v}{N-v}$, then the same conclusion as above holds. Observe that in the special case $v=0,1+\frac{2-v}{N-v}=1+\frac{2}{N}$, which is the Fujita critical exponent [2] for the semilinear heat equation $\frac{\partial u}{\partial t}-\Delta u=u^{p}$. When $k=2$, Mitidieri and Pohozaev [3] obtained the following results for (2)-(4) under suitable conditions and the initial value $u_{1}$. In the case where $v=2$, it was shown that if one of the following assumptions is satisfied: (a) $N \neq 2$ and $1<p \leq 3$; and (b) $N=2$ and $p>1$, then (2)-(4) has no nontrivial global weak solutions. In the case where $v<\min \{2, N\}$, it was shown that if $p(2(N-1)-v) \leq 2(N+1)-3 v$, then the same conclusion as above holds. Observe that in the special case $v=0$, the above condition reduces to $1<p \leq p_{K}^{*}(N)$, where $p_{K}^{*}(N)=\frac{N+1}{N-1}$ $(N \geq 2)$ is the Kato exponent [4] for the hyperbolic inequality $\frac{\partial^{2} u}{\partial t^{2}}-\Delta u \geq|u|^{p}$. In [5], Caristi studied (2)-(4) in the general case where $k \geq 2$. Namely, when $v=2$, it was shown that under suitable conditions for the initial values, if one of the following assumptions is satisfied: (a) $N \neq 2$ and $1<p \leq k+1$; and (b) $N=2$ and $p>1$, then (2)-(4) has no global weak solutions. When $v<2$, it was shown that under suitable conditions on the initial values, if $p(k(N-2)+2-v) \leq N k+2-v(k+1)$, then the same conclusion as above holds.

In the limited case $\alpha \downarrow k$, when $\sigma=0$, (3) reduces to the differential inequality:

$$
\frac{\partial^{k} u}{\partial t^{k}}(t, x)-|x|^{v} \Delta u(t, x) \geq|u(t, x)|^{p}+F(x), \quad(t, x) \in(0, \infty) \times \mathbb{R}^{N} .
$$

In [5], Caristi proved that if $\int_{\mathbb{R}^{N}} F(x)|x|^{-v} d x>0$ and $p(N-2)<N-v$, then (2)-(5) admits no global weak solutions. Observe that the exponent $\frac{N-v}{N-2}$, which appears in the above condition, is the critical exponent for the elliptic inequality (see, e.g., [1]):

$$
-\Delta u \geq|x|^{-v}|u|^{p}, \quad x \in \mathbb{R}^{N} .
$$

Due to the usefulness of fractional derivatives in modeling various phenomena from science and engineering (as can be seen in, e.g., [6-9]), the study of fractional partial differential equations (as well as fractional differential equations) becomes a subject of increasing concern. The study of the nonexistence of global solutions to time-fractional evolution equations and inequalities has been initiated by Kirane and their collaborators (as can be seen in, e.g., [10-14]). In particular, in [13], Kirane et al. studied the nonexistence of nontrivial global weak non-negative solutions to the fractional-in-time and in-space evolution equation:

$$
\frac{\partial^{\alpha} u}{\partial t^{\alpha}}(t, x)+(-\Delta)^{\frac{\beta}{2}} u(t, x)=h(t, x)|u(t, x)|^{p}, \quad(t, x) \in(0, \infty) \times \mathbb{R}^{N},
$$

where $0<\alpha<1,1 \leq \beta \leq 2,(-\Delta)^{\frac{\beta}{2}}$ is the fractional Laplacian of order $\frac{\beta}{2}, p>1$, the function $h$ satisfies $h(t, x) \geq C t^{\rho}|x|^{\sigma}$, and $\rho, \sigma$ satisfy certain conditions. In [15], Zhang and Sun investigated the time-fractional nonlinear diffusion equation:

$$
\frac{\partial^{\alpha} u}{\partial t^{\alpha}}(t, x)-\Delta u(t, x)=|u(t, x)|^{p-1} u(t, x), \quad(t, x) \in(0, \infty) \times \mathbb{R}^{N}
$$

where $0<\alpha<1$ and $p>1$. Namely, under suitable conditions on the initial value $u_{0}$, it was shown that if $1<p<1+\frac{2}{N}$, then any nontrivial non-negative solution to (2)-(6) (with $k=1$ ) blows up in finite time, while if $p \geq 1+\frac{2}{N}$ and $u_{0}$ is sufficiently small with respect to a certain norm, then the problem admits global solutions. For other works related to nonexistence results for fractional evolution equations and inequalities, as can be seen in, e.g., [16-19] and the references therein. 
Motivated by the above contributions, our aim in this paper was to obtain sufficient conditions for which (1)-(2) and (3)-(2) have no global weak solutions in a sense which will be subsequently specified.

The organization of the paper is as follows. In Section 2, we recall the notion of the Caputo fractional derivative and provide some useful lemmas. In Section 3, we define the global weak solutions to (1)-(2) and (3)-(2), and state our main results. In Section 4, we prove Theorem 1. In Section 5, we prove Theorem 2.

\section{Preliminaries}

We refer the reader to [20] for the following definitions.

Let $T>0$ be fixed. We denote by $A C([0, T])$ the space of all real valued functions which are absolutely continuous on $[0, T]$. For a natural number $n \geq 0$, let:

$$
A C^{n+1}([0, T])=\left\{\vartheta:[0, T] \rightarrow \mathbb{R} \mid \vartheta \in C^{n}([0, T]), \frac{d^{n} \vartheta}{d x^{n}} \in A C([0, T])\right\} .
$$

Clearly, we have $A C^{1}([0, T])=A C([0, T])$.

Given $\sigma>0$ and $\vartheta \in L^{1}([0, T])$, the left-sided and right-sided Riemann-Liouville fractional integrals of order $\sigma$ of $\vartheta$ are defined, respectively, by

$$
\left(I_{0}^{\sigma} \vartheta\right)(t)=\frac{1}{\Gamma(\sigma)} \int_{0}^{t}(t-s)^{\sigma-1} \vartheta(s) d s \quad \text { and } \quad\left(I_{T}^{\sigma} \vartheta\right)(t)=\frac{1}{\Gamma(\sigma)} \int_{t}^{T}(s-t)^{\sigma-1} \vartheta(s) d s
$$

for almost everywhere $x \in[0, T]$, where $\Gamma$ denotes the Gamma function.

Given a natural number $k \geq 1, k-1<\alpha<k$, and $\vartheta \in A C^{k}([0, T])$, the (left-sided) Caputo fractional derivative of order $\alpha$ of $\vartheta$ is defined by

$$
\frac{d^{\alpha} \vartheta}{d t^{\alpha}}(t)=\left(I_{0}^{k-\alpha} \vartheta\right)(t)=\frac{1}{\Gamma(k-\alpha)} \int_{0}^{t}(t-s)^{k-\alpha-1} \frac{d^{k} \vartheta}{d t^{k}}(s) d s
$$

for almost everywhere $x \in[0, T]$.

Lemma 1 ([20]). Let $\tau>0, a, b \geq 1$, and $\frac{1}{a}+\frac{1}{b} \leq 1+\tau\left(a \neq 1, b \neq 1\right.$, in the case $\frac{1}{a}+\frac{1}{b}=$ $1+\tau)$. If $(v, w) \in L^{a}([0, T]) \times L^{b}([0, T])$, then:

$$
\int_{0}^{T}\left(I_{0}^{\tau} v\right)(t) w(t) d t=\int_{0}^{T} v(t)\left(I_{T}^{\tau} w\right)(t) d t
$$

The following properties follow from standard calculations (as can be seen in, e.g., [18]).

Lemma 2. For sufficiently large $\lambda$, let:

$$
a_{T}(t)=T^{-\lambda}(T-t)^{\lambda}, \quad t \in[0, T] .
$$

Let $k \geq 1$ be a natural number and $k-1<\alpha<k$. Then:

$$
\begin{aligned}
\left(I_{T}^{k-\alpha} a_{T}\right)(t) & =\frac{\Gamma(\lambda+1)}{\Gamma(k-\alpha+\lambda+1)} T^{-\lambda}(T-t)^{\lambda+k-\alpha}, \\
\frac{d^{i}\left(I_{T}^{k-\alpha} a_{T}\right)}{d t^{i}}(t) & =(-1)^{i} \frac{\Gamma(\lambda+1)}{\Gamma(\lambda+k-\alpha-i+1)} T^{-\lambda}(T-t)^{\lambda+k-\alpha-i}, i=1,2, \cdots, k .
\end{aligned}
$$

\section{Main Results}

$$
\begin{aligned}
\text { Let } \Omega & =[0, \infty) \times \mathbb{R}^{N} \text {. For } T>0, \text { let } \Omega_{T}=[0, T] \times \mathbb{R}^{N} \text { and: } \\
\Phi_{T} & =\left\{\varphi \in C_{t, x}^{k, 2}\left(\Omega_{T}\right): \varphi \geq 0, \varphi \text { is compactly supported with respect to } x\right\} .
\end{aligned}
$$


Using the integration by parts rule given by Lemma 1, we define global weak solutions to (1)-(2) as follows.

Definition 1. We say that $u$ is a global weak solution to (1) if the following conditions are satisfied:

(i) $|u|^{p}|x|^{-v} \in L_{l o c}^{1}(\Omega), u|x|^{-v} \in L_{l o c}^{1}(\Omega), u \in L_{l o c}^{1}(\Omega)$;

(ii) For all $T>0$ and $\varphi \in \Phi_{T}$ :

$$
\begin{aligned}
& \int_{\Omega_{T}}|u|^{p}|x|^{-v} \varphi d x d t \\
& \leq \int_{\Omega_{T}}(-1)^{k} u|x|^{-v} \frac{\partial^{k}\left(I_{T}^{k-\alpha} \varphi\right)}{\partial t^{k}} d x d t-\int_{\Omega_{T}} u \Delta \varphi d x d t \\
& \quad+\sum_{i=0}^{k-1}(-1)^{i+1} \int_{\mathbb{R}^{N}} u_{k-i-1}(x)|x|^{-v} \frac{\partial^{i}\left(I_{T}^{k-\alpha} \varphi\right)}{\partial t^{i}}(0, x) d x
\end{aligned}
$$

Similarly, we define global weak solutions to (3)-(2) as follows.

Definition 2. We say that $u$ is a global weak solution to (3)-(2) if the following conditions are satisfied:

(i) $|u|^{p}|x|^{-v} \in L_{l o c}^{1}(\Omega), u|x|^{-v} \in L_{l o c}^{1}(\Omega), u \in L_{l o c}^{1}(\Omega)$;

(ii) For all $T>0$ and $\varphi \in \Phi_{T}$ :

$$
\begin{aligned}
& \int_{\Omega_{T}}|u|^{p}|x|^{-v} \varphi d x d t+\int_{\Omega_{T}} t^{\sigma} F(x)|x|^{-v} \varphi d x d t \\
& \leq \int_{\Omega_{T}}(-1)^{k} u|x|^{-v} \frac{\partial^{k}\left(I_{T}^{k-\alpha} \varphi\right)}{\partial t^{k}} d x d t-\int_{\Omega_{T}} u \Delta \varphi d x d t \\
& \quad+\sum_{i=0}^{k-1}(-1)^{i+1} \int_{\mathbb{R}^{N}} u_{k-i-1}(x)|x|^{-v} \frac{\partial^{i}\left(I_{T}^{k-\alpha} \varphi\right)}{\partial t^{i}}(0, x) d x
\end{aligned}
$$

For (1)-(2), we have the following nonexistence result.

Theorem 1. Let $u_{i}|x|^{-v} \in L^{1}\left(\mathbb{R}^{N}\right)$ for all $i=0,1, \cdots, k-1$. If:

$$
\int_{\mathbb{R}^{N}} u_{k-1}(x)|x|^{-v} d x>0
$$

and:

$$
p(\alpha(N-2)+(2-v)(1+\alpha-k))<\alpha(N-2)+(2-v)(1+2 \alpha-k)
$$

then (1)-(2) has no global weak solutions.

Remark 1. Observe that, in the limit case where $\alpha \downarrow k,(11)$ reduces to:

$$
p(k(N-2)+2-v)<k N+2-v(k+1)
$$

which is the condition obtained by Caristi [5] (with strict inequality) for which the limit problem (4)-(2) has no global weak solutions.

Then, we consider the inhomogeneous problem (3)-(2). 
Theorem 2. Let $u_{i}|x|^{-v} \in L_{l o c}^{1}\left(\mathbb{R}^{N}\right)$ and $u_{i} \geq 0$, for all $i=0,1, \cdots, k-1$. Assume that $F|x|^{-v} \in L^{1}\left(\mathbb{R}^{N}\right)$ and:

$$
\int_{\mathbb{R}^{N}} F(x)|x|^{-v} d x>0
$$

If:

$$
p(\alpha(N-2)-\sigma(2-v))<\alpha(N-v)-\sigma(2-v)
$$

then (3)-(2) has no global weak solutions.

Remark 2. Observe that in the limit case $\alpha \downarrow k$, when $\sigma=0$, (12) reduces to:

$$
p(N-2)<N-v,
$$

which is the condition obtained by Caristi [5], for which the limit problem (5)-(2) has no global weak solutions.

The proofs of Theorems 1 and 2 are based on the test function method (see, e.g., [1]) and some integral estimates.

\section{Proof of Theorem 1}

We denote by $C$ a positive constant (independent on $T$ ) whose value may change from line to line.

Suppose that $u$ is a global weak solution to (1)-(2). By (8), for sufficiently large $T$, and all $\varphi \in \Phi_{T}$ :

$$
\begin{aligned}
\int_{\Omega_{T}}|u|^{p}|x|^{-v} \varphi d x d t \leq & \int_{\Omega_{T}}|u||x|^{-v}\left|\frac{\partial^{k}\left(I_{T}^{k-\alpha} \varphi\right)}{\partial t^{k}}\right| d x d t+\int_{\Omega_{T}}|u||\Delta \varphi| d x d t \\
& +\sum_{i=0}^{k-1}(-1)^{i+1} \int_{\mathbb{R}^{N}} u_{k-i-1}(x)|x|^{-v} \frac{\partial^{i}\left(I_{T}^{k-\alpha} \varphi\right)}{\partial t^{i}}(0, x) d x .
\end{aligned}
$$

By Young's inequality, we obtain:

$$
\begin{aligned}
\int_{\Omega_{T}}|u||x|^{-v}\left|\frac{\partial^{k}\left(I_{T}^{k-\alpha} \varphi\right)}{\partial t^{k}}\right| d x d t \leq & \frac{1}{2} \int_{\Omega_{T}}|u|^{p}|x|^{-v} \varphi d x d t \\
& +C \int_{\Omega_{T}} \varphi^{\frac{-1}{p-1}}\left|\frac{\partial^{k}\left(I_{T}^{k-\alpha} \varphi\right)}{\partial t^{k}}\right|^{\frac{p}{p-1}}|x|^{-v} d x d t .
\end{aligned}
$$

Similarly, we have:

$$
\int_{\Omega_{T}}|u||\Delta \varphi| d x d t \leq \frac{1}{2} \int_{\Omega_{T}}|u|^{p}|x|^{-v} \varphi d x d t+C \int_{\Omega_{T}} \varphi^{\frac{-1}{p-1}}|\Delta \varphi|^{\frac{p}{p-1}}|x|^{\frac{v}{p-1}} d x d t .
$$

It follows from (13)-(15) that:

$$
\sum_{i=0}^{k-1}(-1)^{i} \int_{\mathbb{R}^{N}} u_{k-i-1}(x)|x|^{-v} \frac{\partial^{i}\left(I_{T}^{k-\alpha} \varphi\right)}{\partial t^{i}}(0, x) d x \leq C\left(I_{1}+I_{2}\right),
$$

where:

$$
I_{1}=\int_{\Omega_{T}} \varphi^{\frac{-1}{p-1}}\left|\frac{\partial^{k}\left(I_{T}^{k-\alpha} \varphi\right)}{\partial t^{k}}\right|^{\frac{p}{p-1}}|x|^{-v} d x d t
$$


and:

$$
I_{2}=\int_{\Omega_{T}} \varphi^{\frac{-1}{p-1}}|\Delta \varphi|^{\frac{p}{p-1}}|x|^{\frac{v}{p-1}} d x d t .
$$

Then, let us consider a cut-off function $\xi \in C_{0}^{\infty}(\mathbb{R})$ satisfying:

$$
0 \leq \xi \leq 1, \quad \xi(s)=\left\{\begin{array}{lll}
1 & \text { if } & 0 \leq s \leq 1 \\
0 & \text { if } & s \geq 2
\end{array}\right.
$$

For sufficiently large $\lambda$ and $\ell$, we take:

$$
\varphi(t, x)=a_{T}(t) b_{T}^{\ell}(x),
$$

where $a_{T}$ is given by (7), and:

$$
b_{T}(x)=\xi\left(\frac{|x|^{2}}{T^{2 \theta}}\right) .
$$

Here, $\theta$ is a positive constant that will be chosen later. We rewrite $I_{1}$ as

$$
I_{1}=\left(\int_{0}^{T} a_{T}^{\frac{-1}{p-1}}(t)\left|\frac{d^{k}\left(I_{T}^{k-\alpha} a_{T}\right)}{d t^{k}}\right|^{\frac{p}{p-1}} d t\right)\left(\int_{\mathbb{R}^{N}} b_{T}^{\ell}(x)|x|^{-v} d x\right)
$$

and $I_{2}$ as

$$
I_{2}=\left(\int_{0}^{T} a_{T}(t) d t\right)\left(\int_{\mathbb{R}^{N}} b_{T}^{\frac{-\ell}{p-1}}\left|\Delta\left(b_{T}^{\ell}\right)\right|^{\frac{p}{p-1}}|x|^{\frac{v}{p-1}} d x\right) .
$$

Through Lemma 2, we have:

$$
\begin{aligned}
\int_{0}^{T} a_{T}^{\frac{-1}{p-1}}(t)\left|\frac{d^{k}\left(I_{T}^{k-\alpha} a_{T}\right)}{d t^{k}}\right|^{\frac{p}{p-1}} d t & =C T^{-\lambda} \int_{0}^{T}(T-t)^{\lambda-\frac{\alpha p}{p-1}} d t \\
& =C T^{1-\frac{\alpha p}{p-1}}
\end{aligned}
$$

Taking in consideration the properties of the function $\xi$ and using that $v<\min \{2, N\}$, we obtain:

$$
\begin{aligned}
\int_{\mathbb{R}^{N}} b_{T}^{\ell}(x)|x|^{-v} d x & =\int_{0<|x|<\sqrt{2} T^{\theta}} \xi^{\ell}\left(\frac{|x|^{2}}{T^{2 \theta}}\right)|x|^{-v} d x \\
& \leq C \int_{r=0}^{\sqrt{2} T^{\theta}} r^{N-1-v} d r \\
& =C T^{\theta(N-v)} .
\end{aligned}
$$

From (20), (22) and (23), we deduce that:

$$
I_{1} \leq C T^{1-\frac{\alpha p}{p-1}+\theta(N-v)} .
$$

Now, let us estimate the term $I_{2}$. First, we have:

$$
\begin{aligned}
\int_{0}^{T} a_{T}(t) d t & =T^{-\lambda} \int_{0}^{T}(T-t)^{\lambda} d t \\
& =C T .
\end{aligned}
$$

On the other hand, by the properties of the function $\xi$, we can easily obtain that:

$$
\left|\Delta\left(b^{\ell}\right)\right| \leq C T^{-2 \theta} \xi^{\ell-2}\left(\frac{|x|^{2}}{T^{2 \theta}}\right), \quad T^{\theta}<|x|<\sqrt{2} T^{\theta} .
$$


Hence (again, due to the properties of the function $\xi$ ), there holds:

$$
\begin{aligned}
\int_{\mathbb{R}^{N}} b_{T}^{\frac{-\ell}{p-1}}\left|\Delta\left(b_{T}^{\ell}\right)\right|^{\frac{p}{p-1}}|x|^{\frac{v}{p-1}} d x & =\int_{T^{\theta}<|x|<\sqrt{2} T^{\theta}} b_{T}^{\frac{-\ell}{p-1}}\left|\Delta\left(b_{T}^{\ell}\right)\right|^{\frac{p}{p-1}}|x|^{\frac{v}{p-1}} d x \\
& \leq C T^{\frac{-2 \theta p}{p-1}} \int_{T^{\theta}<|x|<\sqrt{2} T^{\theta}} \xi^{\ell-\frac{2 p}{p-1}}\left(\frac{|x|^{2}}{T^{2 \theta}}\right)|x|^{\frac{v}{p-1}} d x \\
& \leq C T^{\frac{-2 \theta p}{p-1}} \int_{T^{\theta}<|x|<\sqrt{2} T^{\theta}}|x|^{\frac{v}{p-1}} d x \\
& \leq C T^{\theta\left(\frac{v-2 p}{p-1}+N\right)} .
\end{aligned}
$$

Therefore, it follows from (21), (25) and (26) that:

$$
I_{2} \leq C T^{1+\theta\left(\frac{v-2 p}{p-1}+N\right)} .
$$

Combining (24) with (27), we obtain:

$$
I_{1}+I_{2} \leq C\left(T^{1-\frac{\alpha p}{p-1}+\theta(N-v)}+T^{1+\theta\left(\frac{v-2 p}{p-1}+N\right)}\right) .
$$

Taking $\theta=\frac{\alpha}{2-v}$ (notice that $v<2$ ), the above estimate reduces to:

$$
I_{1}+I_{2} \leq C T^{1-\frac{\alpha p}{p-1}+\frac{\alpha(N-v)}{2-v}} .
$$

On the other hand, by Lemma 2 and the definition of the function $\varphi$, we have:

$$
\begin{aligned}
& \sum_{i=0}^{k-1}(-1)^{i} \int_{\mathbb{R}^{N}} u_{k-i-1}(x)|x|^{-v} \frac{\partial^{i}\left(I_{T}^{k-\alpha} \varphi\right)}{\partial t^{i}}(0, x) d x \\
& =\sum_{i=0}^{k-1}(-1)^{i} \int_{\mathbb{R}^{N}} u_{k-i-1}(x)|x|^{-v} b_{T}^{\ell}(x) \frac{d^{i}\left(I_{T}^{k-\alpha} a_{T}\right)}{d t^{i}}(0) d x \\
& =C \sum_{i=0}^{k-1} T^{k-\alpha-i} \int_{\mathbb{R}^{N}} u_{k-i-1}(x)|x|^{-v} \xi^{\ell}\left(\frac{|x|^{2}}{T^{2 \theta}}\right) d x \\
& =C\left(T^{k-\alpha} \int_{\mathbb{R}^{N}} u_{k-1}(x)|x|^{-v} \xi^{\ell}\left(\frac{|x|^{2}}{T^{2 \theta}}\right) d x+\sum_{i=1}^{k-1} T^{k-\alpha-i} \int_{\mathbb{R}^{N}} u_{k-i-1}(x)|x|^{-v} \mathcal{\zeta}^{\ell}\left(\frac{|x|^{2}}{T^{2 \theta}}\right) d x\right) .
\end{aligned}
$$

Observe that since $u_{i}|x|^{-v} \in L^{1}\left(\mathbb{R}^{N}\right)$ for all $i=0,1, \cdots, k-1$, and due to (10), by the dominated convergence theorem we obtain:

$$
\lim _{T \rightarrow \infty} \int_{\mathbb{R}^{N}} u_{k-1}(x)|x|^{-v} \mathcal{\zeta}^{\ell}\left(\frac{|x|^{2}}{T^{2 \theta}}\right) d x=\int_{\mathbb{R}^{N}} u_{k-1}(x)|x|^{-v} d x>0
$$

and:

$$
\lim _{T \rightarrow \infty} \int_{\mathbb{R}^{N}} u_{k-i-1}(x)|x|^{-v} \xi^{\ell}\left(\frac{|x|^{2}}{T^{2 \theta}}\right) d x=\int_{\mathbb{R}^{N}} u_{k-i-1}(x)|x|^{-v} d x
$$

for all $i=1,2, \cdots, k-1$. Hence, for a sufficiently large $T$, we deduce that:

$$
\begin{aligned}
\sum_{i=0}^{k-1}(-1)^{i} \int_{\mathbb{R}^{N}} u_{k-i-1}(x)|x|^{-v} \frac{\partial^{i}\left(I_{T}^{k-\alpha} \varphi\right)}{\partial t^{i}}(0, x) d x \geq & C T^{k-\alpha} \int_{\mathbb{R}^{N}} u_{k-1}(x)|x|^{-v} d x \\
& +\sum_{i=1}^{k-1} T^{k-\alpha-i} C_{i}
\end{aligned}
$$


where $C_{i} \in \mathbb{R}$ are constants. Then, there holds:

$$
\sum_{i=0}^{k-1}(-1)^{i} \int_{\mathbb{R}^{N}} u_{k-i-1}(x)|x|^{-v} \frac{\partial^{i}\left(I_{T}^{k-\alpha} \varphi\right)}{\partial t^{i}}(0, x) d x \geq C T^{k-\alpha} \int_{\mathbb{R}^{N}} u_{k-1}(x)|x|^{-v} d x .
$$

Then, combining the estimates (16), (28) and (30), we deduce that:

$$
\int_{\mathbb{R}^{N}} u_{k-1}(x)|x|^{-v} d x \leq C T^{1-\frac{\alpha p}{p-1}+\frac{\alpha(N-v)}{2-\nu}+\alpha-k} .
$$

Finally, letting $T \rightarrow \infty$ in the above inequality and using (11), we obtain:

$$
\int_{\mathbb{R}^{N}} u_{k-1}(x)|x|^{-v} d x \leq 0,
$$

which contradicts (10). Thus, we deduce that (1)-(2) admits no global weak solutions. The proof is completed.

\section{Proof of Theorem 2}

Suppose that $u$ is a global weak solution to (3)-(2). By (9), for a sufficiently large $T$, and all $\varphi \in \Phi_{T}$ :

$$
\begin{aligned}
& \int_{\Omega_{T}}|u|^{p}|x|^{-v} \varphi d x d t+\int_{\Omega_{T}} t^{\sigma} F(x)|x|^{-v} \varphi d x d t \\
& \leq \int_{\Omega_{T}}|u||x|^{-v}\left|\frac{\partial^{k}\left(I_{T}^{k-\alpha} \varphi\right)}{\partial t^{k}}\right| d x d t+\int_{\Omega_{T}}|u||\Delta \varphi| d x d t \\
& \quad+\sum_{i=0}^{k-1}(-1)^{i+1} \int_{\mathbb{R}^{N}} u_{k-i-1}(x)|x|^{-v} \frac{\partial^{i}\left(I_{T}^{k-\alpha} \varphi\right)}{\partial t^{i}}(0, x) d x .
\end{aligned}
$$

Using (31), the estimates (14) and (15), there holds:

$$
\sum_{i=0}^{k-1}(-1)^{i} \int_{\mathbb{R}^{N}} u_{k-i-1}(x)|x|^{-v} \frac{\partial^{i}\left(I_{T}^{k-\alpha} \varphi\right)}{\partial t^{i}}(0, x) d x+\int_{\Omega_{T}} t^{\sigma} F(x)|x|^{-v} \varphi d x d t \leq C\left(I_{1}+I_{2}\right),
$$

where $I_{1}$ and $I_{2}$ are given, respectively, by (17) and (18). Let $\varphi$ be the test function given by (19). Since $u_{i} \geq 0$ for all $i=0,1, \cdots, k-1$, by (29) and (32), we deduce that:

$$
\int_{\Omega_{T}} t^{\sigma} F(x)|x|^{-v} \varphi d x d t \leq C\left(I_{1}+I_{2}\right) .
$$

On the other hand, by the definition of the function $\varphi$, we have:

$$
\int_{\Omega_{T}} t^{\sigma} F(x)|x|^{-v} \varphi d x d t=\left(\int_{0}^{T} t^{\sigma} a_{T}(t) d t\right)\left(\int_{\mathbb{R}^{N}} F(x)|x|^{-v} b_{T}^{\ell}(x) d x\right) .
$$

By the definition of the function $a_{T}$, there holds:

$$
\begin{aligned}
\int_{0}^{T} t^{\sigma} a_{T}(t) d t & =T^{-\lambda} \int_{0}^{T} t^{\sigma}(T-t)^{\lambda} d t \\
& \geq T^{-\lambda} \int_{0}^{\frac{T}{2}} t^{\sigma}(T-t)^{\lambda} d t \\
& \geq \frac{C}{\sigma+1} T^{\sigma+1} .
\end{aligned}
$$


Moreover, by the definitions of the functions $b_{T}$ and $\xi$, since $F|x|^{-v} \in L^{1}\left(\mathbb{R}^{N}\right)$, the dominant convergence theorem yields:

$$
\lim _{T \rightarrow \infty} \int_{\mathbb{R}^{N}} F(x)|x|^{-v} b_{T}^{\ell}(x) d x=\lim _{T \rightarrow \infty} \int_{\mathbb{R}^{N}} F(x)|x|^{-v} \mathcal{\xi}^{\ell}\left(\frac{|x|^{2}}{T^{2 \theta}}\right) d x=\int_{\mathbb{R}^{N}} F(x)|x|^{-v} d x .
$$

Since $\int_{\mathbb{R}^{N}} F(x)|x|^{-v} d x>0$, for sufficiently large $T$, we have:

$$
\int_{\mathbb{R}^{N}} F(x)|x|^{-v} b_{T}^{\ell}(x) d x \geq C \int_{\mathbb{R}^{N}} F(x)|x|^{-v} d x .
$$

Thus, it follows from (34)-(36) that:

$$
\int_{\Omega_{T}} t^{\sigma} F(x)|x|^{-v} \varphi d x d t \geq C T^{\sigma+1} \int_{\mathbb{R}^{N}} F(x)|x|^{-v} d x .
$$

Then, by (33) and (37), we deduce that:

$$
\int_{\mathbb{R}^{N}} F(x)|x|^{-v} d x \leq C T^{-\sigma-1}\left(I_{1}+I_{2}\right) .
$$

Taking $\theta=\frac{\alpha}{2-v}$ and using the estimate (28), we obtain:

$$
\int_{\mathbb{R}^{N}} F(x)|x|^{-v} d x \leq C T^{-\sigma-\frac{\alpha p}{p-1}+\frac{\alpha(N-v)}{2-v}} .
$$

Finally, using (12), and letting $T \rightarrow \infty$ into the above inequality, we obtain $\int_{\mathbb{R}^{N}} F(x)|x|^{-v} d x \leq 0$, which contradicts the condition $\int_{\mathbb{R}^{N}} F(x)|x|^{-v} d x>0$. The proof of Theorem 2 is completed.

\section{Conclusions}

In this paper, the nonexistence of global weak solutions to the fractional-in-time evolution inequalities (1)-(2) and (3)-(2) was investigated. Using the test function method and some integral estimates, sufficient conditions depending on the parameters of the problems were obtained so that there were no global weak solutions. Namely, for (1)-(2), under suitable conditions for the initial values, we proved that (see Theorem 1) if:

$$
p(\alpha(N-2)+(2-v)(1+\alpha-k))<\alpha(N-2)+(2-v)(1+2 \alpha-k),
$$

then (1)-(2) has no global weak solutions. For (3)-(2), under suitable conditions for the initial values and the inhomogeneous term $F(x)$, we proved that (see Theorem 2) if:

$$
p(\alpha(N-2)-\sigma(2-v))<\alpha(N-v)-\sigma(2-v)
$$

then (3)-(2) has no global weak solutions. In the limit case $\alpha \downarrow k$, we recovered some known results from the literature.

This contribution only deals with time-fractional evolution inequalities with subcritical degeneracy $(v<2)$. It will also be interesting to study time-fractional evolution inequalities with critical degeneracy. Namely, the problems (1)-(2) and (3)-(2) with $v=2$.

Author Contributions: Formal analysis, R.P.A.; Investigation, S.M.A., M.J. and B.S. All authors equally contributed to this paper. All authors have read and agreed to the published version of the manuscript.

Funding: The third author is supported by the Researchers Supporting Project number (RSP-2021/57), King Saud University, Riyadh, Saudi Arabia.

Institutional Review Board Statement: Not applicable. 
Informed Consent Statement: Not applicable.

Data Availability Statement: Not applicable.

Conflicts of Interest: The authors declare no conflict of interest.

\section{References}

1. Mitidieri, E.; Pohozaev, S.I. Nonexistence of weak solutions for some degenerate elliptic and parabolic problems on $\mathbb{R}^{N}$. J. Evol. Equ. 2001, 1, 189-220. [CrossRef]

2. Fujita, H. On the blowing up of solutions of the Cauchy problem for $u_{t}=\Delta u+u^{\alpha+1}$. J. Fac. Sci. Univ. Tokyo Sect. 1996, 13, 109-124.

3. Mitidieri, E.; Pohozaev, S.I. Nonexistence of weak solutions for degenerate and singular hyperbolic problems on $\mathbb{R}^{N}$. Proc. Steklov Inst. Math. 2000, 232, 1-19.

4. Kato, T. Blow-up of solutions of some nonlinear hyperbolic equations. Commun. Pure Appl. Math. 1980, 33, 501-505. [CrossRef]

5. Caristi, G. Nonexistence of global solutions of higher order evolution inequalities in $\mathbb{R}^{N}$. In Nonlinear Equations: Methods, Models and Applications, Progress in Nonlinear Differential Equations and Their Applications; Lupo, D., Pagani, C.D., Ruf, B., Eds.; Birkhäuser: Basel, Switzerland, 2003; Volume 54, pp. 91-105.

6. Meng, R. Application of fractional calculus to modeling the non-linear behaviors of ferroelectric polymer composites: viscoelasticity and dielectricity. Membranes 2021, 11, 409. [CrossRef] [PubMed]

7. Sun, H.; Li, R.; Xu, J.; Xu, F.; Zhang, B.; Dong, X. Fractional modeling and characteristic analysis of hydro-pneumatic suspension for construction vehicles. Processes 2021, 9, 1414. [CrossRef]

8. Lazopoulos, A.K.; Karaoulanis, D. On $\Lambda$-fractional viscoelastic models. Axioms 2021, 10, 22. [CrossRef]

9. Alcántara-López, F.; Fuentes, C.; Chávez, C.; Brambila-Paz, F.; Quevedo, A. Fractional growth model applied to COVID-19 data. Mathematics 2021, 9, 1915. [CrossRef]

10. Fino, A.Z.; Kirane, M. Qualitative properties of solutions to a time-space fractional evolution equation. Q. Appl. Math. 2012, 70, 133-157. [CrossRef]

11. Kirane, M.; Ahmad, B.; Alsaedi, A.; Al-Yami, M. Non-existence of global solutions to a system of fractional diffusion equations. Acta Appl. Math. 2014, 133, 235-248. [CrossRef]

12. Kirane, M.; Laskri, Y. Nonexistence of global solutions to a hyperbolic equation with a space-time fractional damping. Appl. Math Comput. 2005, 167, 1304-1310. [CrossRef]

13. Kirane, M.; Laskri, Y.; Tatar, N.E. Critical exponents of Fujita type for certain evolution equations and systems with spatio-temporal fractional derivatives. J. Math. Anal. Appl. 2005, 312, 488-501. [CrossRef]

14. Kirane, M.; Nabti, A. Life span of solutions to a nonlocal in time nonlinear fractional Schrödinger equation. Z. Angew. Math. Phys. 2015, 66, 1473-1482. [CrossRef]

15. Zhang, Q.G.; Sun, H.R. The blow-up and global existence of solutions of Cauchy problems for a time fractional diffusion equation. Topol. Methods Nonlinear Anal. 2015, 46, 69-92. [CrossRef]

16. Jleli, M.; Samet, B.; Vetro, C. On a fractional in time nonlinear Schrödinger equation with dispersion parameter and absorption coefficient. Symmetry 2020, 12, 1197. [CrossRef]

17. Jleli, M.; Samet, B.; Vetro, C. Large time behavior for inhomogeneous damped wave equations with nonlinear memory. Symmetry 2020, 12, 1609. [CrossRef]

18. Jleli, M.; Samet, B.; Vetro, C. Nonexistence results for higher order fractional differential inequalities with nonlinearities involving Caputo fractional derivative. Mathematics 2021, 9, 1866. [CrossRef]

19. Zhang, Q.; Sun, H.R.; Li, Y. The nonexistence of global solutions for a time fractional nonlinear Schrödinger equation without gauge invariance. Appl. Math. Lett. 2017, 64, 119-124. [CrossRef]

20. Kilbas, A.A.; Srivastava, H.M.; Trujillo, J.J. Theory and Applications of Fractional Differential Equations; North-Holland Mathematical Studies; Elsevier (North-Holland) Science Publishers: Amsterdam, The Netherlands; London, UK; New York, NY, USA, 2006; Volume 204. 